\title{
CELLULAR COVERS OF LOCAL GROUPS
}

\author{
RAMÓN FLORES AND JÉRÔME SCHERER
}

\begin{abstract}
We prove that, in the category of groups, the composition of a cellularization and a localization functor need not be idempotent. This provides a negative answer to a question of Emmanuel Dror Farjoun.
\end{abstract}

\section{INTRODUCTION}

Cellularization and localization functors are idempotent functors that are respectively augmented and coaugmented. The interest for a systematic study of such functors comes from Homotopy Theory, mainly through early work of Bousfield, [5], and Farjoun's more recent book [9]. The names themselves are designed so as to remind us of cell complexes and p-local spaces or modules. In Group Theory, localization functors appear explicitly and in full generality in Casacuberta's [6, Section 3], but many examples, such as abelianization or localization at a set of primes are classical. Likewise, cellularization functors for groups appear relatively late and are described for the first time in [20], even though specific cellular constructions such as universal central extensions have played an important role in Group Theory.

It is helpful to think about localization and cellularization as functors that act on the whole category of groups, transforming some groups drastically, possibly killing many of them, and allowing us to focus on some special features such as torsion or divisibility. In [4] the authors studied the effect of iterating different cellularization functors on a given (finite) group, that is, looking at the "orbit" of a group under the action of all possible cellularization functors. A similar approach in the coaugmented case is probably quite difficult, as shown by the work of Rodríguez and Scevenels, [19].

2000 Mathematics Subject Classification. Primary 20F99; Secondary 55P60, 20E22, 20F50, $55 \mathrm{R} 35$.

This work was supported by the Spanish Ministry of Education and Science MEC-FEDER grant MTM2010-15831 and MTM2016-80439-P, and the Andalusian government under grants FQM-213 and P07-FQM-2863. 
The aim of this note is to analyze the effect of iterating a cellularization and a localization in the category of groups. Once a group $G$ has been transformed by a localization functor $G \rightarrow L G$, and after it has been modified by a cellularization functor cell $L G \rightarrow L G$, it seems that the group cell $L G$ is frequently left unchanged by further application of this procedure. In this case cell $L G$ is a fixed point in the category of groups for the composition cell $\circ L$. As we recall below in Section 2, all known examples provide such fixed points and it is in fact a question Farjoun asked in [1, Question 3] whether cell $\circ L$ and $L \circ$ cell are idempotent functors, without specifying the underlying category.

The first author gave a negative answer to this question in the case of homotopy localization and cellularization functors of spaces, [12]. His counterexample cannot be adapted in the category of groups as it uses in a central way the flexibility of having homotopy groups in different dimensions. We have been wondering since then how to attack this problem for groups, one major obstruction being the difficulty to perform explicit computations. Our theorem is based on a very recent computation, [13], of a certain cellularization of large Burnside groups. The specific form of this cellularization is the key to the unexpected behaviour of the iteration of the two functors we choose. It is rooted in the work of many mathematicians who provided negative answers to the Burnside problem, in particular Ol'shanskiü's intriguing computation of the Schur multiplier of Burnside groups at large primes, [17. Corollary 31.2], see also Adian and Atabekyan's improved bound in [3].

We conclude this introduction by recalling that any group theoretical example involving cellularization and localization can be upgraded to a homotopical example via the classifying space functor. Thus, our counterexample provides a new counterexample to Farjoun's question in the category of spaces, simpler than the original proof in 12 in that all spaces are $K(\pi, 1)$ 's, but more subtle in that it relies on the restricted Burnside problem.

Acknowledgments. We thank Antonio Viruel, Delaram Kahrobaei and Simon Smith for helpful conversations. We also thank Varujan Atabekyan and Alexander $\mathrm{Yu}$. Olshanskii for helping us out with the second homology group of Burnside groups. The first author wishes to thank the École Polytechnique Fédérale de Lausanne for its kind hospitality when this joint project started. 


\section{BACKGROUND ON LOCALIZATION AND CELLULARIZATION}

We will work mostly in the category of groups and will thus simply refer to localization and cellularization functors without specifying that they are functors of groups. If necessary, we will make clear when we deal with space valued functors by calling them homotopical localization and homotopical cellularization functors.

A localization functor $L$ is an idempotent and coaugmented functor. The coaugmentation is traditionally written $\eta_{G}: G \rightarrow L G$ and idempotency means that both $\eta_{L G}$ and $L \eta_{G}$ are isomorphisms for any group $G$. Typical localization functors are obtained by "inverting" a group homomorphism $\varphi: A \rightarrow B$. A group $G$ is $\varphi$-local if $\operatorname{Hom}(\varphi, G)$ is an isomorphism and a group homomorphism $\psi$ is a $\varphi$-local equivalence if $\operatorname{Hom}(\psi, G)$ is an isomorphism for any $\varphi$-local group $G$. Localization with respect to $\varphi$ is the localization functor $L_{\varphi}$ which is characterized by the fact that the coaugmentation $\eta_{G}: G \rightarrow L_{\varphi} G$ is a $\varphi$-local equivalence to a $\varphi$-local group.

Example 1.1. Our main player in the world of localization functors is reduction $\bmod p$, i.e. localization with respect to the epimorphism $\varphi: \mathbb{Z} \rightarrow \mathbb{Z} / p$, which, with the multiplicative notation, is the morphism $\varphi: F_{1} \rightarrow C_{p}$ from the free group on one generator to the cyclic group of order $p$, sending the generator to a chosen generator. We will write $L_{p}$ for this functor from now on. Loosely speaking, the effect of $L_{p}$ on a group $G$ is to kill $q$-torsion for $q \neq p$ and to convert elements of order $p^{n}$ or of infinite order into order $p$ elements. In other words $L_{p}$ is left adjoint to the inclusion in the category of groups of the variety of groups of exponent $p$.

A cellularization functor cell is an idempotent and augmented functor. The augmentation is traditionally written $\epsilon_{G}: \operatorname{cell} G \rightarrow G$. The only known cellularization functors arise as follows. Choose a group $A$ and define a group homomorphism $\psi$ to be an $A$-cellular equivalence if $\operatorname{Hom}(A, \psi)$ is an isomorphism. A group $G$ is $A$ cellular if $\operatorname{Hom}(G, \psi)$ is an isomorphism for any $A$-cellular equivalence $\psi$. Cellular groups are characterized by the property that they belong to $\mathcal{C}(A)$, the smallest class containing $A$ and closed under isomorphisms and arbitrary colimits, [20, Section 3].

Example 1.2. Our object of interest in the world of cellularization functors is $C_{p^{-}}$ cellularization, which we will write $\operatorname{cell}_{p}$ for short. Loosely speaking $\operatorname{cell}_{p} G \rightarrow G$ is the best approximation of $G$ that can be constructed out of cyclic groups of order $p$. 
We mention next the construction by Casacuberta and Descheemaeker of relative group completions, [7, Theorem 3.2]. We will call it fiberwise group localization, which uses the name of the homotopical analogue, [9, Theorem 1.F.1].

Theorem 1.3 (Casacuberta-Descheemaeker). Let $A \longmapsto B \rightarrow C$ be an extension of groups, and let $L$ be a localization functor. Then there exists a commutative diagram

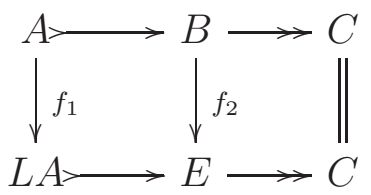

such that $f_{1}$ is L-localization and $f_{2}$ is an L-local equivalence.

The methods we use in this article are mostly of a group theoretical nature. There are however situations where homotopy theory provides helpful tools. The first one is about so-called nullification functors, i.e. localization with respect to a map of the form $A \rightarrow *$. The standard notation for such a functor is $P_{A}$. In group theory, the morphism $G \rightarrow P_{A} G$ is an epimorphism to the largest quotient of $G$ such that $\operatorname{Hom}(A, G)$ is trivial, [6, Theorem 3.2]. In homotopy theory $A$ is a space and $X \rightarrow P_{A} X$ is obtained from $X$, up to homotopy, by taking the homotopy cofiber $X_{1}$ of the map $\bigvee \Sigma^{k} A \rightarrow X$, where the wedge is taken over representatives of all homotopy classes of maps out of suspensions of $A$, for $k \geq 0$, and by repeating this procedure. The nullification $P_{A} X$ is the homotopy colimit of the telescope $X=X_{0} \rightarrow X_{1} \rightarrow X_{2} \ldots$, see for example [8, Section 17]. We will use the relationship between the group theoretical nullification with respect to $C_{p}$ and the homotopical nullification with respect to the Moore space $M\left(C_{p}, 1\right)=S^{1} \cup_{p} e^{2}$, as well as the analogous relation between group theoretical and homotopical cellularization.

Proposition 1.4. Let $G$ be any group. Then

(1) $\pi_{1} P_{M\left(C_{p}, 1\right)} K(G, 1) \cong P_{C_{p}} G$;

(2) $\pi_{1} \operatorname{cell}_{M\left(C_{p}, 1\right)} K(G, 1) \cong \operatorname{cell}_{p} G$.

The first isomorphism is [6, Theorem 3.5] and the second one is [20, Theorem 3.3]. In order to identify the space $\operatorname{cell}_{M\left(C_{p}, 1\right)} K(G, 1)$ we will use Chachólski's fibration from [8, Theorem 20.3]. We start with the cofibration sequence

$$
\bigvee M\left(C_{p}, 1\right) \rightarrow K(B, 1) \rightarrow C o f
$$


where the wedge is taken over representatives of all homotopy classes from $M\left(C_{p}, 1\right)$ to $K(B, 1)$. The Moore space $M\left(C_{p}, 2\right)$ is the suspension of $M\left(C_{p}, 1\right)$

Theorem 1.5 (Chachólski). The cellularization cell ${ }_{M\left(C_{p}, 1\right)} K(G, 1)$ is weakly equivalent to the homotopy fiber of the composite map $K(G, 1) \rightarrow \operatorname{Cof} \rightarrow P_{M\left(C_{p}, 2\right)}$ Cof.

\section{IDEMPotent EXAMPLES}

This section is devoted to describe examples where the composition of a cellularization and a localization functor is an idempotent functor. Note that the composite will not be, in general, augmented nor coaugmented.

Example 2.1. Let $p$ be a prime number. Consider the cellularization functor cell $_{p}$ introduced above, and $P_{p}$ is nullification with respect to $C_{p}$, i.e. localization with respect to the constant map $C_{p} \rightarrow\{1\}$. The effect of cell $_{p}$ is described in [20, Theorem 3.7], while, for any group $G, P_{p} G$ is isomorphic to the quotient of $G$ by its $p$-radical $T_{p}(G)$, which is the largest $p$-torsion free quotient of $G$.

The cellularization cell $_{p} G$ belongs to the class of $C_{p}$-cellular groups $\mathcal{C}\left(C_{p}\right)$, which is contained in the class $\overline{\mathcal{C}\left(C_{p}\right)}$ of $C_{p}$-acyclic groups, [20, Section 4] (this class consists by definition in all groups $G$ that are "killed" by $C_{p}$, i.e. $\left.P_{p} G=\{1\}\right)$. In particular $P_{p} c e l l_{p} G=\{1\}$. Likewise, as there are no non-trivial homomorphisms from $C_{p}$ to $P_{p} G$, the group $\operatorname{cell}_{p} P_{p} G$ is also trivial. So in this case both functors $P_{p} c e l l_{p}$ and cell $_{p} P_{p}$ are idempotent.

Example 2.2. Consider now two distinct primes $p$ and $q$, and assume furthermore that $G$ is finite. In this case $\operatorname{cell}_{p} G$ has been described in [11, Lemma 4.5] as an extension

$$
K \rightarrow \operatorname{cell}_{p} G \rightarrow S_{p} G,
$$

where $S_{p} G$ is the subgroup of $G$ generated by its order $p$ elements, and $K$ is the quotient of $H_{2}\left(S_{p} G ; \mathbb{Z}\right)$ by its $p$-torsion subgroup. We compute $\operatorname{cell}_{p} P_{q} G$. As $P_{q} G=$ $G / T_{q} G, q$ does not divide the order of $P_{q} G$, and the same happens to $\operatorname{cell}_{p} P_{q} G$ as can be seen from the previous extension. Thus, there is an isomorphism $\operatorname{cell}_{p} P_{q} G \cong$ $P_{q}$ cell $_{p} P_{q} G$, so that the latter is a $C_{p}$-cellular group. This implies that $\operatorname{cell}_{p} P_{q}$ is idempotent when applied to $G$.

This is an instance of a very frequent phenomenon, in which some cellular deformation of a local group turns out to be local again, or, as illustrated in our next 
example, some localization of a cellular group is cellular. Both cases imply idempotency in the composition.

Example 2.3. We wish here to understand $P_{q}$ cell $l_{p}$ for two distinct primes $p$ and $q$. Let $G$ be a finite group. We will prove that $P_{q} c e l l_{p} G$ is cellular.

From the extension in Example 2.2, as $G$ is finite, it follows that we only need to check that $P_{q}$ cell $G$ is generated by order $p$ elements, and that $H_{2}\left(P_{q} c e l l_{p} G ; \mathbb{Z}\right)$ is $p$-torsion. The former is clear, as $P_{q} \operatorname{cell} l_{p} G$ is a quotient of $c e l l_{p} G$, a finite group generated by elements of order $p$.

To prove the latter, observe that $P_{q} \operatorname{cell} l_{p} G$ is $q$-torsion free, hence so is the second homology group $H_{2}\left(P_{q} \operatorname{cell}_{p} G ; \mathbb{Z}\right)$. We use now Proposition 1.4 (1) to identify $P_{q} \operatorname{cell}_{p} G$ with the fundamental group of $P_{M\left(C_{q}, 1\right)} K\left(\operatorname{cell}_{p} G, 1\right)$. This space is obtained by killing successively all maps out of the Moore space $M\left(C_{q}, 1\right)$ and its suspensions. The homology groups of the homotopy cofibers $X_{0}, X_{1}, X_{2}$, etc., described above fit thus in an exact sequence

$$
H_{2}\left(X_{k} ; \mathbb{Z}\right) \rightarrow H_{2}\left(X_{k+1} ; \mathbb{Z}\right) \rightarrow H_{1}\left(\bigvee \Sigma^{k} M\left(C_{q}, 1\right) ; \mathbb{Z}\right)
$$

Since $H_{2}\left(X_{0} ; \mathbb{Z}\right)$ is $p$-torsion - because $X_{0}=K\left(\operatorname{cell}_{p} G, 1\right)$ - and the homology of the Moore space $M\left(C_{q}, 1\right)$ is $q$-torsion, this proves by induction that the second homology group $H_{2}\left(P_{M\left(C_{q}, 1\right)} K\left(\operatorname{cell}_{p} G, 1\right) ; \mathbb{Z}\right)$ is an abelian group having only $p$ - and $q$-torsion.

Let us call $X=P_{M\left(C_{q}, 1\right)} K\left(\operatorname{cell}_{p} G, 1\right)$, a space whose fundamental group is isomorphic to $P_{q}$ cell $_{p} G$ and consider the universal covering fibration sequence

$$
\tilde{X} \rightarrow X \rightarrow K\left(P_{q} \operatorname{cell}_{p} G, 1\right)
$$

An easy Serre spectral sequence argument shows that $H_{2}\left(P_{q} \operatorname{cell}_{p} G ; \mathbb{Z}\right)$ is a quotient of $H_{2}(X ; \mathbb{Z})$ (this phenomenon had been already observed by Hopf, [14]). This second homology group must thus be $p$-torsion, which shows that $P_{q} c e l l_{p} G$ is $C_{p}$-cellular. Hence $P_{q} \operatorname{cell}_{p} P_{q}$ cell $_{p} G=P_{q}$ cell $_{p} G$.

Example 2.4. Often LcellG is the trivial group. This happened in Example 2.1, and also for example when $G$ is a finite simple group, $L=L_{a b}$ is abelianization (localization with respect to the homomorphism $\mathbb{Z} * \mathbb{Z} \rightarrow \mathbb{Z} \oplus \mathbb{Z}$ ) and cell $G$ is any cellular cover of $G$. This comes from the fact that cell $G$ is a perfect group, 4, Section 11]. 
Example 2.5. Let $L=L_{a b}$ be abelianization. Since any cellularization functor transforms an abelian group into an abelian group, [10, Theorem 1.4], cell $L_{a b} G$ is an abelian group which is thus both cellular and $L_{a b}$-local.

Example 2.6. The inclusion $i: A_{5} \hookrightarrow A_{6}$ is a localization by [22, Section 3(i)]. The group $A_{5}$ is $C_{2}$-cellular since it is generated by elements of order 2 and $H_{2}\left(A_{5} ; \mathbb{Z}\right) \cong$ $\mathbb{Z} / 2$.

We choose thus cell $l_{2}$ and $L_{i}$ so that $L_{i} c e l l_{2} A_{5}=A_{6}$. However $A_{6}$ is not $C_{2}$-cellular because $H_{2}\left(A_{6} ; \mathbb{Z}\right) \cong \mathbb{Z} / 6$. In fact cell $_{2} A_{6}$ must be a central extension with prime to $p$ torsion center, so it is $3 . A_{6}$, the Valentiner group, a triple cover of $A_{6}$. This group is not $i$-local as it contains $A_{5}$, but not $A_{6}$ (the central extension is not split). It is very likely that $L_{i}\left(3 . A_{6}\right)$ is $A_{6}$, but we have not found a proof this fact.

\section{Cellularizing the Burnside group}

Let $p$ be a prime and let $B$ be the Burnside group $B_{2, p}$, which is the quotient of a free group $F_{2}$ on two generators by the normal subgroup generated by all $p$-th powers. Thus $B=L_{p} F_{2}$ is free in the variety of groups of exponent $p$. Alternatively, and this is our starting point for the next computations, let $F$ be the free product $C_{p} * C_{p}$ of two copies of cyclic groups of order $p$. This is a $C_{p}$-cellular group and $B \cong L_{p} F$ as $F$ is obtained by adding to the free group $F_{2}$ only relations which are exponent $p$ reduction. We have thus $L_{p} \operatorname{cell}_{p} F \cong B$.

The computation we present in this section is that of $\operatorname{cell}_{p} B$, for a sufficiently large prime. By this we mean that $p \geq 665$, i.e. $p$ is a prime at least equal to 671 . We rely on Ol'shanskiı's work in [17] where $p>10^{10}$, or rather on the improved bound $p \geq 665$ in Adian and Atabekyan's [3]. Our next proposition is a particular case of the much more general statement [13, Proposition 3.9]. We identify $\operatorname{cell}_{p} B$ by elementary methods for the sake of completeness, using in a crucial way the strong link between homotopical and group theoretical cellularization.

Proposition 3.1. [13, Proposition 5.2] Let $p$ be sufficiently large. The $C_{p}$-cellular approximation of $B$ fits in a central extension

$$
K \longmapsto \operatorname{cell}_{p} B \rightarrow B
$$

where $\mathrm{K}$ is isomorphic to $\mathrm{H}_{2}(B ; \mathbb{Z})$, the Schur multiplier of $B$, a free abelian group in a countable number of generators. 
Proof. We have seen in Proposition $1.4(2)$ that $\operatorname{cell}_{p} B$ is isomorphic to the fundamental group of $\operatorname{cell}_{M\left(C_{p}, 1\right)} K(B, 1)$. Our goal is thus to compute this cellularization of the Eilenberg-Mac Lane space $K(B, 1)$ (in the category of pointed spaces). For this purpose we use Chachólski's Theorem 1.5 and start with the cofibration sequence

$$
\bigvee M\left(C_{p}, 1\right) \rightarrow K(B, 1) \rightarrow C o f
$$

where the wedge is taken over representatives of all homotopy classes from $M\left(C_{p}, 1\right)$ to $K(B, 1)$. The cellularization $\operatorname{cell}_{M\left(C_{p}, 1\right)} K(B, 1)$ is then the homotopy fiber of the composite map $K(B, 1) \rightarrow C o f \rightarrow P_{M\left(C_{p}, 2\right)}$ Cof.

Since $B$ is generated by elements of order $p$, the cofiber $C o f$ is simply connected and $H_{2}(C o f ; \mathbb{Z})$ is an extension of a direct sum $\oplus \mathbb{Z} / p$ by $H_{2}(B ; \mathbb{Z})$. The latter is a free abelian group in a countable number of generators by [17, Corollary 31.2] or [3], this is where we use that $p$ is large. When nullifying with respect to the simply connected space $M\left(C_{p}, 2\right)=S^{2} \cup_{p} e^{3}$ one kills the $p$-torsion in $H_{2}(C o f ; \mathbb{Z})$ so that only a free abelian group is left in $H_{2}\left(P_{M\left(C_{p}, 2\right)} \operatorname{Cof} ; \mathbb{Z}\right) \cong \pi_{2} P_{M\left(C_{p}, 2\right)}$ Cof. The homotopy long exact sequence associated to Chachólski's fibration sequence cell $_{M\left(C_{p}, 1\right)} K(B, 1) \rightarrow K(B, 1) \rightarrow P_{M\left(C_{p}, 2\right)}$ Cof yields an extension

$$
\pi_{2} P_{M\left(C_{p}, 2\right)} \text { Cof } \longmapsto \pi_{1} \operatorname{cell} l_{M\left(C_{p}, 1\right)} K(B, 1) \rightarrow B
$$

which allows us to conclude.

Remark 3.2. When $p=2$, the Burnside group $B=C_{2} \times C_{2}$ is $C_{2}$-cellular, i.e. cell $l_{2} B=B$. At the prime $3, B$ is an extraspecial nilpotent group of class 2 of order $3^{3}$, hence $C_{3}$-cellular since $H_{2}(B ; \mathbb{Z})$ is 3 -torsion, see [11, Proposition 4.8]. At the prime 5 it is not known whether $B$ is finite and we do not know what $\operatorname{cell}_{p} B$ looks like at a "small" prime $5 \leq p<671$.

\section{Localizing The CELlularized Burnside Group}

From now on we will denote $\operatorname{cell}_{p} B$ by $C$ and $p$ is a sufficiently large prime. In this section we compute $L_{p} C$ and the next lemma will help us on the way.

Lemma 4.1. The fiberwise $L_{p}$-localization of the extension $C$ is a central extension $L_{p} K \longmapsto E \rightarrow B$. 
Proof. If we apply fibrewise group localization (Theorem 1.3) to the extension in Proposition 3.1, we obtain a commutative diagram of homomorphisms:

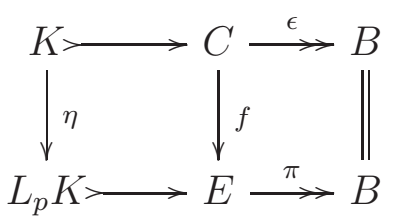

We observe that the coaugmentation $\eta: K \rightarrow L_{p} K$ is exponent $p$ reduction, i.e. $L_{p} K \cong \oplus \mathbb{Z} / p$ is a countable $\mathbb{F}_{p}$-vector space. We deduce then from the Five Lemma that $f: C \rightarrow E$ is an epimorphism, which sends by naturality the center of $C$ to the center of $E$. The Burnside group $B$ is known to be centerless by [2, Theorem VI.3.4], so that $Z(C)=K$. Therefore $L_{p} K$ is contained in $Z(E)$, in other words the extension $L_{p} K \longmapsto E \rightarrow B$ is central.

Proposition 4.2. Let $E$ be the fiberwise $L_{p}$-localization of the extension $C$. Then $E$ is a group of exponent $p$.

Proof. As $L_{f} K$ and $B$ are groups of exponent $p, E$ is a group whose non-trivial elements can have order $p$ and $p^{2}$. We should check that the latter is impossible. Let $x$ be any element in $E$ and set $b=\pi(x)$. As $C=$ cell $_{p} B$ is the $C_{p}$-cellularization of $B$, the augmentation induces a bijective map

$$
\epsilon_{*}: \operatorname{Hom}\left(C_{p}, C\right) \cong \operatorname{Hom}\left(C_{p}, B\right)
$$

and this guarantees that there exists a preimage $c \in \epsilon^{-1}(b)$ of order $p$ (or one if $b=1$ ). By commutativity of the diagram in the proof of Lemma 4.1, $\pi(x)=\epsilon(c)=\pi(f(c))$, and hence $x=f(c) t$ for a certain element $t \in L_{p} K$. Since $t$ belongs to $L_{f} K$, a group of exponent $p$, both $c$ and $t$ have order (at most) $p$. Moreover $t$ is central in $E$ so that $x^{p}=(f(c) t)^{p}=f(c)^{p} t^{p}=1$, and we are done.

Corollary 4.3. The extension $E$ is the $L_{p}$-localization of $C$.

Proof. Because of Theorem 1.3, the map $f: C \rightarrow E$ is an $L_{p}$-equivalence. By the previous proposition, $E$ is $L_{p}$-local, and then $L_{p} C \cong E$.

In fact, we can say more about the structure of $E$.

Proposition 4.4. The extension $E$ splits as a direct product $E \cong L_{p} K \times B$. 
Proof. We will prove that the map $\pi: E \rightarrow B$ has a section. As the extension is central by Lemma 4.1, it must then split as a direct product.

The Burnside group $B$ is free, in the variety of groups of exponent $p$, on two generators $\bar{x}$ and $\bar{y}$. Let us choose preimages $x$ and $y$ in $E$. Remember that $B$ is $L_{p} F_{2}$ and call $a$ and $b$ two generators of the free group $F_{2}$ that are taken by the coaugmentation $\eta: F_{2} \rightarrow B$ to $\bar{x}$ and $\bar{y}$ respectively.

Since $F_{2}$ is free, there exists a unique lift $q: F_{2} \rightarrow E$ (taking $a$ to $x$ and $b$ to $y$ ). By Proposition 4.2 $E$ is a group of exponent $p$, hence $q$ factors uniquely through a homomorphism $g: B \rightarrow E$. This means that the composite

$$
\pi \circ g \circ \eta: F_{2} \rightarrow B \rightarrow E \rightarrow B
$$

coincides with the coaugmentation $\eta$. The universal property of localization shows then that $\pi \circ g$ is the identity, i.e. $g$ is a section of $\pi$.

Remark 4.5. Note that a similar splitting cannot exist for the extension that defines $C$. If it did exist, this would imply that the free abelian group $K$ would be a retract of a $C_{p}$-cellular space, hence $C_{p}$-cellular itself.

Now we have produced one of our two desired counterexamples.

Theorem 4.6. The groups $L_{p}$ cell $F$ and $L_{p}$ cell $L_{p} L_{p}$ cell $F$ are not isomorphic.

Proof. We have proved that $L_{p} c e l l_{p} F$ is the Burnside group $B$, which is centerless. On the other hand, Proposition 4.4 implies that $L_{p} c e l l_{p} L_{p} c e l l_{p} F=E$ has (many) order $p$ elements in the center.

\section{Cellularizing one step further}

The next result is an easy consequence of Proposition 4.4.

Proposition 5.1. We have an isomorphism cell s $_{p} \cong L_{p} K \times C$.

Proof. The cellularization of a product is the product of the cellularizations, see for example [10, Lemma 2.3(1)]. We have then $\operatorname{cell}_{p} E \cong \operatorname{cell}_{p}\left(L_{p} K \times B\right) \cong L_{p} K \times C$ because $L_{p} K$ is $C_{p}$-cellular.

From this we obtain our second counterexample.

Theorem 5.2. The groups cell $L_{p} B$ and cell $L_{p}$ cell $_{p} L_{p} B$ are not isomorphic. 
Proof. The Burnside group $B$ is $L_{p}$-local and $\operatorname{cell}_{p} L_{p} B$ is the group $C$. The group $\operatorname{cell}_{p} L_{p} \operatorname{cell}_{p} L_{p} B=\operatorname{cell}_{p} L_{p} C=\operatorname{cell}_{p} E$ is the direct product $L_{p} K \times C$ as we have established in Proposition 5.1. This group has uncountably many order $p$ elements in the center whereas the center of $C$ is the free abelian group $K$.

We have been trying out quite a few localization and cellularization functors before coming up with the above computation. Let us mention a few promising ones, which we have not been able to fully understand. Libman showed that the inclusion $A_{n} \hookrightarrow A_{n+1}$ is a localization (with respect to the inclusion itself), [15, Example 3.4], and the universal central extension 2. $A_{n+1} \rightarrow A_{n+1}$ is a cellular cover by [4, Section 11]. Libman also found examples of infinite localizations of finite groups, [16], and Przeździecki showed they can be arbitrarily large, [18]. Iterating this procedure is not manageable, but if we wish to understand the dynamics of the effect of idempotent functors on the category of groups it would be important to make progress in this direction.

To conclude we would like to address a natural question.

Question 5.3. Is there a cellularization functor cell, a localization functor $L$, and an abelian group $A$ such that LcellLcell $A \neq L$ cell $A$ or cellLcellLA $\neq$ cellLA?

We believe that the answer to Farjoun's question is negative as well in the category

of abelian groups. It is tempting to use the inclusion $\bigoplus_{n} \mathbb{Z} / p^{n} \hookrightarrow \prod_{n} \mathbb{Z} / p^{n}$, which is a localization by [21, Example 6.5], but we have not found an adequate cellularization functor to continue.

\section{REFERENCES}

1. Guido's book of conjectures, Enseign. Math. (2) 54 (2008), no. 1-2, 3-189, A gift to Guido Mislin on the occasion of his retirement from ETHZ, June 2006, Collected by Indira Chatterji. MR 2454697

2. S. I. Adian, The Burnside problem and identities in groups, Ergebnisse der Mathematik und ihrer Grenzgebiete [Results in Mathematics and Related Areas], vol. 95, Springer-Verlag, Berlin-New York, 1979, Translated from the Russian by John Lennox and James Wiegold. MR 537580

3. S. I. Adian and V. Atabekyan, Central extensions of free periodic groups of odd period $n \geq 665$, English translation to appear in Math. Notes.

4. M. Blomgren, W. Chachólski, E. D. Farjoun, and Y. Segev, Idempotent transformations of finite groups, Adv. Math. 233 (2013), 56-86. MR 2995665 
5. A. K. Bousfield, Constructions of factorization systems in categories, J. Pure Appl. Algebra 9 (1976/77), no. 2, 207-220.

6. C. Casacuberta, Anderson localization from a modern point of view, The Čech centennial (Boston, MA, 1993), Contemp. Math., vol. 181, Amer. Math. Soc., Providence, RI, 1995, pp. 35-44. MR 1320986

7. C. Casacuberta and A. Descheemaeker, Relative group completions, J. Algebra 285 (2005), no. 2, 451-469. MR 2125447

8. W. Chachólski, On the functors $C W_{A}$ and $P_{A}$, Duke Math. J. 84 (1996), no. 3, 599-631.

9. E. Dror Farjoun, Cellular spaces, null spaces and homotopy localization, Lecture Notes in Mathematics, vol. 1622, Springer-Verlag, Berlin, 1996.

10. E. Dror Farjoun, R. Göbel, and Y. Segev, Cellular covers of groups, J. Pure Appl. Algebra 208 (2007), no. 1, 61-76. MR 2269828

11. R. Flores, Nullification and cellularization of classifying spaces of finite groups, Trans. Amer. Math. Soc. 359 (2007), no. 4, 1791-1816. MR 2272149

12. _ On the idempotency of some composite functors, Israel J. Math. 187 (2012), 81-91. MR 2891699

13. R. Flores and F. Muro, Torsion homology and cellular approximation, preprint available on the MathArXiV: 1707.07654.

14. H. Hopf, Fundamentalgruppe und zweite Bettische Gruppe, Comment. Math. Helv. 14 (1942), 257-309. MR 0006510

15. A. Libman, Cardinality and nilpotency of localizations of groups and G-modules, Israel J. Math. 117 (2000), 221-237. MR 1760593

16. _ A note on the localization of finite groups, J. Pure Appl. Algebra 148 (2000), no. 3, 271-274. MR 1758733

17. A. Yu. Ol'shanskiı̌, Geometry of defining relations in groups, Mathematics and its Applications (Soviet Series), vol. 70, Kluwer Academic Publishers Group, Dordrecht, 1991, Translated from the 1989 Russian original by Yu. A. Bakhturin. MR 1191619

18. A. J. Przeździecki, Large localizations of finite groups, J. Algebra 320 (2008), no. 12, 4270-4280. MR 2464105

19. J. L. Rodríguez and D. Scevenels, Iterating series of localization functors, Une d $\mathrm{A} @$ coustation topologique [Topological morsels]: homotopy theory in the Swiss Alps (Arolla, 1999), Contemp. Math., vol. 265, Amer. Math. Soc., Providence, RI, 2000, pp. 211-221. MR MR1804680

20. J. L. Rodríguez and J. Scherer, Cellular approximations using Moore spaces, Cohomological methods in homotopy theory (Bellaterra, 1998), Progr. Math., vol. 196, Birkhäuser, Basel, 2001, pp. 357-374.

21. J. L. Rodríguez, J. Scherer, and L. Strüngmann, On localization of torsion abelian groups, Fund. Math. 183 (2004), no. 2, 123-138. MR 2127962

22. J. L. Rodríguez, J. Scherer, and J. Thévenaz, Finite simple groups and localization, Israel J. Math. 131 (2002), 185-202. MR 1942308 

Ramón Flores
Jérôme Scherer
Departamento de Geometría y Topología
Institute of Mathematics
Universidad de Sevilla
EPFL, Station 8
41080 - Sevilla, Spain
CH - 1015 Lausanne, Switzerland
E-mail: ramonjflores@us.es
E-mail: jerome.scherer@epfl.ch 